\title{
Supporting vulnerable physicians at high risk from COVID-19 during the pandemic: a call for action
}

\author{
Mandeep Singh, MBBS MD MSc (i) - Dolores McKeen, MD MSc
}

Received: 20 December 2020/Revised: 26 January 2021 / Accepted: 31 January 2021/Published online: 11 March 2021

(C) Canadian Anesthesiologists' Society 2021

Because of its high infectivity and risks for devastating sequalae, coronavirus disease (COVID-19) caused by the severe acute respiratory syndrome coronavirus 2 (SARSCoV-2) continues to be a challenge for healthcare workers (HCWs). ${ }^{1}$ The potentially overwhelming burden of disease also greatly stresses healthcare system capacity and requires extraordinary organizational planning. Anesthesiologists are particularly vulnerable to the risk of viral transmission that can occur through aerosol and droplet transmission, and less commonly from contaminated surfaces, ${ }^{2}$ as was seen in the previous severe acute respiratory syndrome outbreak. ${ }^{3}$ Airway management that anesthesiologists and others perform requires close contact with infected patients and risks viral

\footnotetext{
M. Singh, MBBS MD MSc ( $\varangle)$

Department of Anesthesiology and Pain Management, Women's

College Hospital, Toronto, ON, Canada

e-mail: mandeep.singh@uhn.ca
}

Department of Anesthesiology and Pain Management, Toronto Western Hospital, University Health Network, University of Toronto, 399 Bathurst Street, McL 2-405, Toronto, ON M5T 2S8, Canada

Department of Anesthesiology and Pain Medicine, University of Toronto, Toronto, Canada

D. McKeen, MD MSc

Department of Anesthesiology and Pain Medicine, University of Toronto, Toronto, Canada

Department of Anesthesia, IWK Health Centre, Halifax, NS, Canada

Department of Anesthesia, Dalhousie University, Halifax, NS, Canada exposure during aerosol-generating medical procedures such as endotracheal intubation and extubation. ${ }^{4}$

Initial efforts during the COVID-19 pandemic were largely targeted at organizational planning for infection prevention and control (IPAC) training and availability of personal protective equipment (PPE). ${ }^{5,6}$ Nevertheless, many HCWs have conditions that elevate their risk for severe complications or death if they develop COVID-19. Concrete recommendations addressing these $\mathrm{HCW}$ vulnerabilities, and increasing measures for protection, have been lacking. ${ }^{7}$

Recommendations from the Government of Canada identifying vulnerable populations were directed at the general population, and did not specifically address protection of $\mathrm{HCWs}$ at elevated risk. $^{8}$ Vulnerable populations have generally been defined as individuals at risk of more severe COVID-19-related adverse outcomes due to pre-existing conditions including advanced age (i.e., increasing risk with each decade, especially $>60 \mathrm{yr}$ of age); other chronic medical conditions (e.g., lung disease, heart disease, high blood pressure, diabetes, kidney disease, liver disease, stroke, or dementia); immunocompromised states, including those with an underlying medical condition (e.g., cancer, human immunodeficiency virus infection) or when taking medications that impair the immune system (e.g., chemotherapy, steroids); and obesity (body mass index $\geq$ $40 \mathrm{~kg} \cdot \mathrm{m}^{28}$

In the United Kingdom (UK), the "extremely clinically vulnerable" were identified as those in the community who have a specific set of conditions, ${ }^{9}$ including solid organ transplant recipients, specific cancers, severe respiratory conditions, people with rare diseases at risk of infection, those on certain immunosuppressive therapies, and concomitant pregnancy with immune suppression or heart 


\section{Provincial and National}

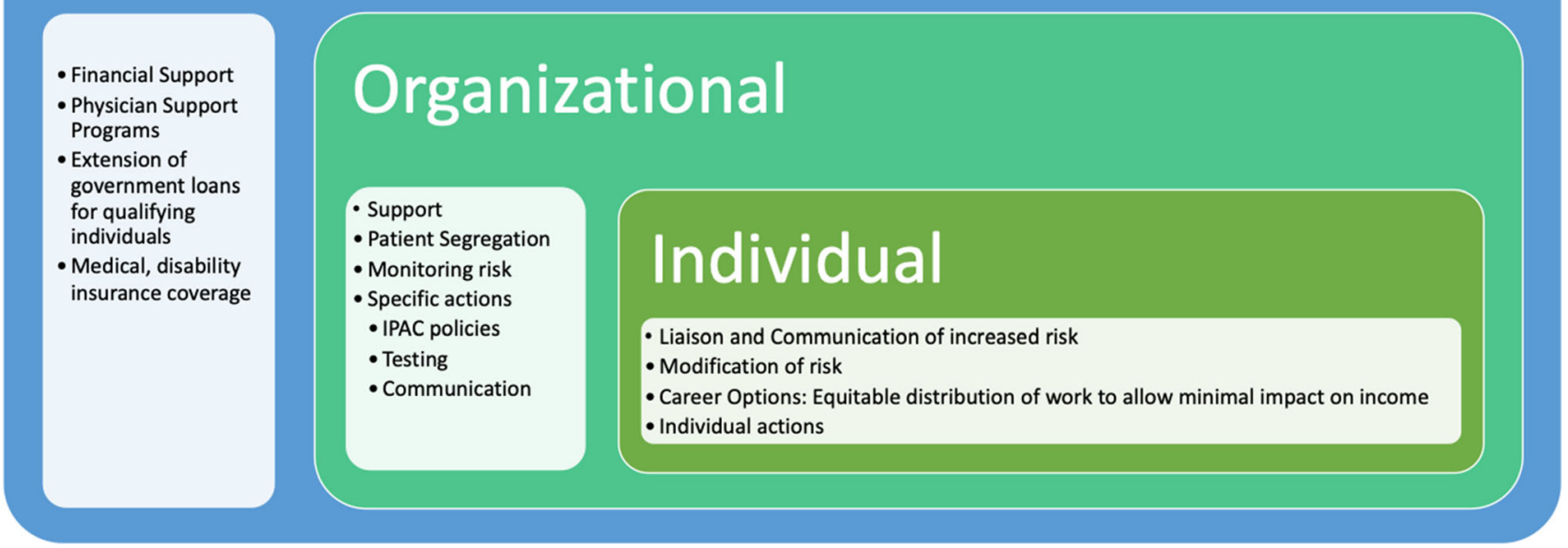

FIGURE Proposed framework and strategies

disease. Additionally, HCWs considered to be extremely clinically vulnerable were issued with clear instructions from the UK government to shield themselves from highrisk healthcare situations, with the National Health Service (NHS) identifying these physicians as needing "workplace shielding". Shielding refers to the extra level of precautions to be followed by persons deemed clinically extremely vulnerable to infection from SARS-CoV-2. ${ }^{10}$ Shielding instructions were stringent and were in place for many months during the first COVID-19 wave. Some physicians were also shielding "by proxy"-i.e., to protect another adult or child within their own household.

A public survey in the UK of physicians who were shielding revealed that the largest group $(55 / 116 ; 47 \%)$ were anesthesiologists and critical care physicians. ${ }^{11}$ Ninety-one percent $(106 / 116)$ were shielding because of their own health condition, with $9 \%$ shielding by proxy (10/116). Fifty-two percent (60/116) of respondents were following the generic advice from government, while others were following specific advice from their Occupational Health Department or other local risk assessment protocol. Immunosuppression, respiratory conditions, and pregnancy were the most common medical reasons for physician shielding. ${ }^{11}$

\section{Shielding in the Canadian context}

Vulnerable physicians comprise a significant proportion of the Canadian physician workforce-e.g., physicians $>65$ $\mathrm{yr}$ of age comprise $16 \%$ of the overall physician workforce, including $15 \%$ of all specialists, and $13 \%$ of anesthesiologists. $^{12,13}$ Canadian data for SARS-CoV-2 infection rates among $\mathrm{HCWs}$ indicates a variability across the provinces; however, detailed data on physician vs non-physician infections, and across medical specialties has been lacking. ${ }^{14}$ Quebec and Ontario had the highest HCW infection rates (24.1\%, and $16.7 \%$, respectively), and all $12 \mathrm{HCW}$ deaths in Canada. ${ }^{14}$ Canadian physicians, particularly anesthesiologists and critical care specialists, should be afforded guidance on the issue of shielding during this pandemic in the Canadian setting. The Canadian Anesthesiologists' Society (CAS) position statement on Physician Shielding During A Pandemic, ${ }^{15}$ endorsed by the Canadian Medical Association (CMA), ${ }^{16}$ should be considered a "call to action" supporting vulnerable physicians at high-risk from COVID-19.

During the initial peak of the pandemic in March-June 2020, some vulnerable older anesthesiologists chose to stop participating in on-call duties, entered early retirement, took sabbatical leave, or took unpaid leave from work. ${ }^{A}$ Nevertheless, vulnerable physicians in younger age groups (e.g., those raising a family) and/or those facing significant debt from earlier educational costs, faced significant pressure to continue working to support their financial situations. As the second wave of the pandemic is currently underway during the winter season in Canada (and with potential subsequent waves also looming), this group of physicians may have to endure another wave of stress and uncertainty.

\footnotetext{
A Multiple personal communciations between colleagues, departmental leadership meetings, and Ontario Medical Association Townhall Meetings during the COVID-19 pandemic.
} 
The CAS recognizes that anesthesiologists have an ethical duty to strive to stay healthy. ${ }^{17}$ Both the CMA's Code of Ethics ${ }^{18}$ and the American Society of Anesthesiologists' Guidelines for the Ethical Practice of Anesthesiology ${ }^{19}$ emphasize that anesthesiologists have ethical responsibilities to themselves, and that the practice of quality anesthesia care requires that they optimally maintain their own health and wellbeing. ${ }^{19}$

We advocate for the following strategies for shielding of vulnerable Canadian anesthesiologists and critical care physicians (Table, FIGURE). ${ }^{15}$ The call to action of the CAS position statement on shielding should empower governments, institutions, and departments to both support and advocate for vulnerable anesthesiologists at the front lines of COVID-19 care, and indeed the overall health and wellbeing of every physician. Individualized personalized risk assessments should be made as well as organizational level assessments to prevent increased risk of infection, harm, and death from COVID-19. ${ }^{20-22}$ Institutional and departmental considerations should be made for equitable distribution of work, financial support, and access to provision of safe clinical care. This includes prioritization to receive vaccination, ${ }^{23,24}$ as well as increasing options where feasible for remote access and virtual care for patients as their colleagues perform other high-risk clinical work. Institutions should promote income stability programs to support anesthesiologists and other HCWs in avoiding any major financfial penalty incumbent with changes in practice patterns and/or scope of practice due to any of the aforenoted pandemic restrictions and/or cancellation of routine perioperative care. For individuals shielding by proxy, institutions should provide a flexible working schedule that considers roles as physicians, parents, and caregivers because these individuals may

TABLE Specific measures for vulnerable physicians during the COVID-19 pandemic

\begin{tabular}{|c|c|}
\hline Strategy & Action \\
\hline \multicolumn{2}{|l|}{ Vulnerable physicians } \\
\hline $\begin{array}{l}\text { Liaison and communication } \\
\text { of risk }\end{array}$ & Departmental and university leadership, occupational health, and human resources. \\
\hline $\begin{array}{l}\text { Modification of personal } \\
\text { health risk }\end{array}$ & $\begin{array}{l}\text {-Identification as per the latest data, local or worldwide. } \\
\text { - Regular follow-up with their treating physician to discuss optimization of their health condition and risk } \\
\text { management strategies. } \\
\text {-Seek support for mental wellbeing, and financial support structure. }\end{array}$ \\
\hline Career options & $\begin{array}{l}\text { - Returning to work in low-risk settings e.g., virtual care, elective surgeries, off-loading other colleagues who are } \\
\text { working in COVID-19 settings, to balance clinical work requirements and equitable distribution of income. } \\
\text {-Working from home, prolonged leave, or a sabbatical. } \\
\text { - Consider change in specialty, or pre-mature retirement for older or financially secure physicians. }\end{array}$ \\
\hline Individual actions & $\begin{array}{l}\text { Use virtual or remote patient contact as much as possible. } \\
\text { Use remote internal meetings when practical. } \\
\text { Use of appropriate PPE, social distancing measures, and compliance with local IPAC measures. }\end{array}$ \\
\hline
\end{tabular}

\section{Organizational level}

Pillar of support

Patient segregation

Monitoring risk

Specific actions
Vulnerability to physical harm also increases vulnerability to mental health issues and feelings of isolation and moral injury. Organizations should provide all the necessary support for these individuals to allow for equitable distribution of income opportunities.

Prioritize contact with COVID-19 negative and asymptomatic patients for vulnerable physicians.

Prevent and eliminate contact with COVID-19 positive or COVID-19 suspected patients.

Including community-, in-hospital-, and individual transmission of infection and adverse outcomes.

Prioritization of vaccination programs to provide immunity against COVID-19.

Vulnerable physicians should not be made to practice clinical care directly or indirectly for COVID-19 positive or COVID-19 suspected patients.

Effectively segregate patients by extent of risk of COVID-19.

Test patients and staff sufficiently to understand the dynamics of COVID-19 and exposure prevalence in hospital. Ensure strict and continuous adherence to face mask policies by staff and patients at all times.

Maintain strict standard IPAC measures.

\section{Provincial and national level}

Financial support Creation of physician support programs to provide support during the peak of the current and future pandemics. 
need to be off work as a result of the loss of childcare (e.g., due to a sick or exposed caregiver at home, elderly parent caregiver, school or daycare closure) or added exposure/ quarantine/testing for the children.

\section{Shielding challenges}

Facing unexpected challenges to their personal safety and disruption to careers and training, UK physicians found themselves confined to their homes longer than then their counterparts, with no control on when and how to return to work. ${ }^{25}$ This disconnection from the frontline created significant practical and psychological challenges. ${ }^{25}$ Issues related to voluntary disclosure of their medical condition with colleagues subjected them to potential stigmatization and moral injury from feeling not able to contribute to the pandemic response in the same capacity as their colleagues (e.g., not taking "protected code blue" or intubation team calls). This exposed them to additional mental and sleep health disruptions further impacting their wellbeing. ${ }^{26-29}$

\section{Equity and financial concerns}

Limiting the scope of work or returning to work requires coordinated planning and agreement from national bodies, institutions, local departments, and the returnee. ${ }^{10}$ Both the UK NHS and Canadian healthcare settings are publicly funded, yet have certain differences that have become apparent on this issue. ${ }^{30}$ The cohesive response of UK physicians resulted in a more unified response to create support systems for the shielding physician and provided a supportive environment for the trainees and consultant physicians, both of which served to minimize impact on their training and salary. The British Medical Association positioned that no doctor should be financially penalized for not being able to undertake certain types of work due to risks posed to their health. ${ }^{10}$

While this support was seemingly well communicated, a great deal of variability existed in individual's experiences of risk assessments and support. Reports emerged of doctors being pressured into working in inappropriate, and vulnerable on-call environments, along with employers seeking ways not to honour payments, despite the fact individuals continued to be clinically vulnerable. ${ }^{10}$

Compared with the UK NHS, Canada's single-payer layer of financing is highly decentralized (e.g., provincially administered) in terms of service delivery. In addition, Canadian physicians are most commonly independent contractors, billing provincial health plans on a fee-forservice basis. ${ }^{31}$ There is limited accountability in the relationship between physicians and governments, health authorities, or hospitals, despite the fact that physicians work within the boundaries of regional or provincial health authorities. This is partly because the hospitals often have separate budgets and independent boards, and decisionmaking is often fragmented. ${ }^{32}$ This environment makes it challenging for Canadian anesthesiologists to request financial and other support for shielding.

Efforts directed towards supporting vulnerable physicians and HCWs should be balanced against the safety and protection of those anesthesiologists in lower risk categories, such that protection of all anesthesia staff and learners is kept confidential, performed in an equitable, non-discriminatory fashion, and does not compromise patient safety (Table, FIGURE). We acknowledge that these measures may be additionally challenging in the current Canadian anesthesiology work force shortage. ${ }^{33,34}$ Importantly, the ethical principles described in the CAS position statement also apply to the broader healthcare workforce; HCWs in long-term care homes and hospital ward settings are also at infectious risk. This is related to a multitude of factors such as working in patientfacing $v s$ non-patient-facing roles, clinical $v s$ non-clinical roles (e.g., housekeeping, portering), ${ }^{35}$ occupational $v s$ household/community exposure, working at single or multiple locations, availability of PPE, and adherence to IPAC measures. ${ }^{36,37}$ While attention is currently focused on the care of those with COVID-19, there are still co-existing healthcare needs including the need to resume routine surgical care that put a strain on our healthcare resources and HCWs. Recognizing our collective ethical duty to the public good, we must take care to minimize risks to our healthcare teams and direct the vulnerable physicians and HCWs to settings at lower risk of infection.

In summary, the call to action of the CMA-endorsed CAS position statement on Physician Shielding During A Pandemic $^{15,16}$ provides an ethical framework and strategies to healthcare systems and institutions to address the concerns of vulnerable physicians, trainees, and other HCWs. By advocating in partnership with national agencies such as the CMA and the Canadian Centre for Occupational Health and Safety (CCOHS), ${ }^{38}$ national standards for shielding HCWs should be established. A national effort headed by the CAS, in collaboration with the CMA and the CCOHS, should be undertaken to collect data on the number of anesthesia providers who have been infected and, where known, the circumstances of the infection (e.g., during patient care $v s$ elsewhere in hospital/community). We advocate that physicians and all HCWs be supported to not only feel safe and protected from direct injury but also be supported morally by their own departmental and institutional leadership. A variety of accommodations should be made available that include options for remote training and development, comprehensive risk assessment, and individualized plans for resuming full scope and/or return to work and training. 


\section{Soutenons les médecins vulnérables à haut risque pendant la pandémie de COVID-19 : agissons maintenant!}

En raison de son infectiosité élevée et des importants risques de séquelles dévastatrices, la maladie du coronavirus (COVID-19) causée par le syndrome respiratoire aigu sévère du coronavirus 2 (SRAS-CoV-2) demeure un défi pour les travailleurs de la santé (TS). ${ }^{1}$ Le fardeau potentiellement écrasant de la maladie met également à l'épreuve la capacité du système de soins de santé et nécessite une planification organisationnelle exceptionnelle. Les anesthésiologistes sont particulièrement vulnérables au risque de transmission virale qui peut survenir par la transmission d'aérosols et de gouttelettes, moins fréquemment par des surfaces contaminées, ${ }^{2}$ comme ce fut le cas lors de l'épidémie précédente de syndrome respiratoire aigu sévère. ${ }^{3}$ La prise en charge des voies aériennes que les anesthésiologistes et d'autres réalisent nécessite un contact rapproché avec les patients infectés et augmente le risque d'exposition virale lors d'interventions médicales générant des aérosols telles que l'intubation et l'extubation des voies respiratoires. ${ }^{4}$ (FIGURE).

Les premiers efforts déployés pendant la pandémie de COVID-19 visaient principalement la planification organisationnelle de la formation sur la prévention et le contrôle des infections (PCI) et la disponibilité des équipements de protection individuelle (EPI). ${ }^{5,6}$
Néanmoins, de nombreux TS ont des problèmes de santé qui augmentent leur risque de complications graves ou de décès s'ils sont touchés par la COVID-19. Il n'y a pas eu de recommandations concrètes concernant les TS plus vulnérables, ni concernant une augmentation des mesures de protection pour ces personnes. ${ }^{7}$

Les recommandations du gouvernement du Canada visant à identifier les populations vulnérables s'adressaient à la population générale et ne traitaient pas spécifiquement de la protection des TS courant un risque élevé. ${ }^{8}$ Les populations vulnérables ont généralement été définies comme les personnes courant un risque de complications liées au COVID-19 plus graves en raison de problèmes de santé préexistants, y compris un âge avancé (soit un risque augmentant avec chaque décennie d'âge, en particulier au-dessus de 60 ans); d'autres atteintes médicales chroniques (p. ex., maladie pulmonaire, maladie cardiaque, hypertension artérielle, diabète, maladie rénale, maladie hépatique, accident vasculaire cérébral ou démence); les états immunovulnérables, y compris ceux accompagnés d'un problème de santé médical sous-jacent (p. ex., cancer, infection au virus de l'immunodéficience humaine) ou la prise de médicaments qui diminuent le système immunitaire (p. ex., chimiothérapie, stéroïdes); et l'obésité (indice de masse corporelle $\geq 40 \mathrm{~kg} \cdot \mathrm{m}^{2}$ ). ${ }^{8}$

$\mathrm{Au}$ Royaume-Uni, les individus « extrêmement vulnérables cliniquement » ont été identifiés comme étant les personnes de la collectivité présentant un ensemble spécifique de problèmes de santé,,$^{9}$ y compris les receveurs de greffes d'organes solides, les personnes atteintes de certains cancers spécifiques ou d'affections respiratoires sévères, celles atteintes de maladies rares à risque

\section{Provincial and National}

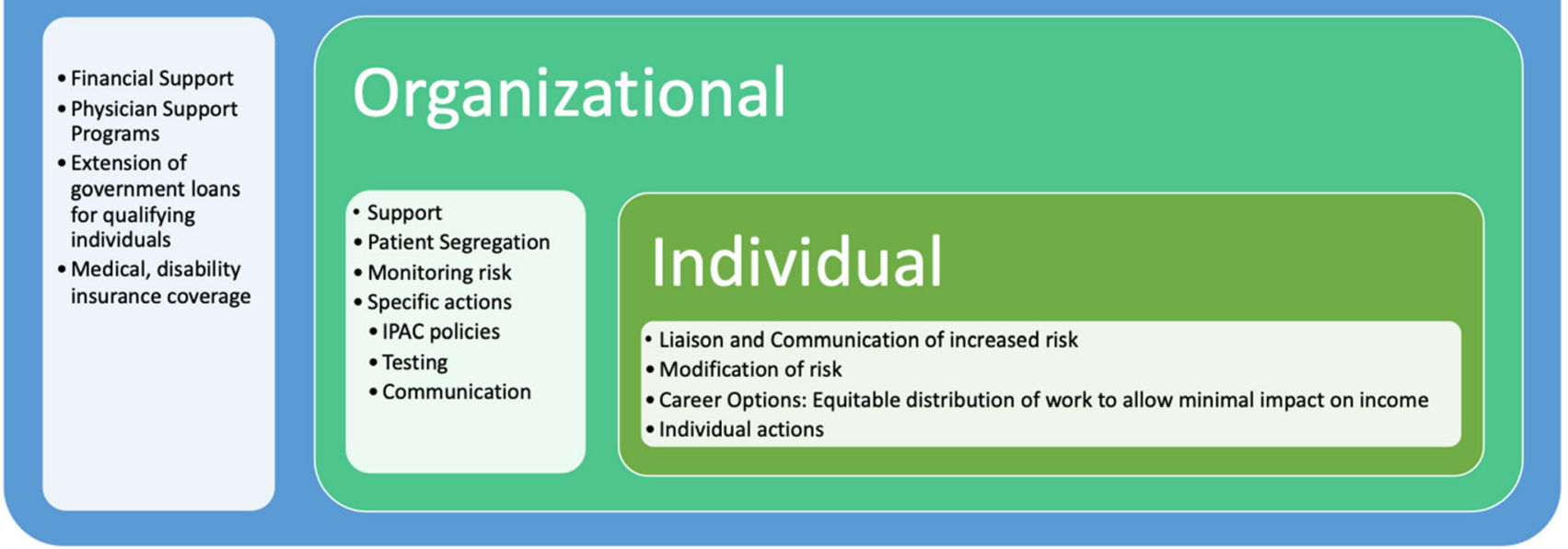

FIGURE Cadre et stratégies proposés 
d'infection ou qui prennent certaines thérapies immunosuppressives ainsi que les personnes vivant une grossesse concomitante avec suppression immunitaire ou maladie cardiaque. En outre, les TS considérés comme étant extrêmement vulnérables cliniquement ont reçu des instructions claires du gouvernement britannique afin qu'ils se protègent des situations de soins de santé à haut risque, le National Health Service (NHS) identifiant ces médecins comme ayant besoin d'une «protection sur le lieu de travail ». Cette protection (« shielding » en anglais) fait référence aux précautions supplémentaires à observer par les personnes jugées extrêmement vulnérables cliniquement à une infection par le SRAS-CoV-2. ${ }^{10}$ Les instructions de protection étaient rigoureuses et ont été maintenues pendant de nombreux mois au cours de la première vague de COVID-19. Certains médecins se protégeaient également pour causes « de proximité », c'est-à-dire pour protéger un autre adulte ou un autre enfant au sein de leur foyer.

Une enquête publique menée au Royaume-Uni auprès de médecins qui bénéficiaient de cette protection a révélé que le groupe le plus nombreux $(55 / 116 ; 47 \%)$ était composé des anesthésiologistes et des intensivistes. ${ }^{11}$ Quatre-vingt-onze pour cent (106/116) utilisaient cette protection en raison de leur propre état de santé, et $9 \%$ s'en servaient comme protection de proximité (10/116). Cinquante-deux pour cent $(60 / 116)$ des répondants suivaient les conseils du gouvernement, tandis que d'autres suivaient les conseils spécifiques de leur département d'hygiène au travail ou un autre protocole local d'évaluation des risques. L'immunosuppression, les maladies respiratoires et la grossesse étaient les raisons médicales les plus fréquentes de protection chez les médecins. ${ }^{11}$

\section{La protection dans le contexte canadien}

Les médecins vulnérables représentent une proportion importante des médecins canadiens. Par exemple, les médecins de plus de 65 ans représentent $16 \%$ de l'ensemble des médecins, dont $15 \%$ de tous les spécialistes et $13 \%$ des anesthésiologistes. ${ }^{12,13}$ Les données canadiennes concernant les taux d'infection par le SRAS-CoV-2 chez les TS indiquent une variabilité d'une province à l'autre; toutefois, des données détaillées sur les infections chez les médecins vs les non-médecins, et par spécialité médicale, font défaut. ${ }^{14}$ Le Québec et l'Ontario ont connu les taux d'infection les plus élevés chez les TS (24,1\% et $16,7 \%$, respectivement), et les 12 décès de TS rapportés au Canada. ${ }^{14}$ Les médecins canadiens, en particulier les anesthésiologistes et les intensivistes, devraient être accompagnés dans cette question de protection pendant cette pandémie. L'énoncé de position de la Société canadienne des anesthésiologistes (SCA) sur la Protection des médecins en période de pandémie, ${ }^{15}$ endossé par l'Association médicale canadienne (AMC), ${ }^{16}$ devrait être considéré comme un " appel à l'action » en soutien aux médecins vulnérables à haut risque de COVID-19.

$\mathrm{Au}$ cours du premier pic de la pandémie, en mars-juin 2020, certains anesthésiologistes vulnérables plus âgés ont choisi d'arrêter de faire de la garde, de prendre une retraite anticipée, un congé sabbatique ou un congé sans solde. ${ }^{\mathrm{A}}$ Toutefois, les médecins vulnérables des groupes d'âge plus jeunes (p. ex., les personnes ayant une famille à charge) et/ ou celles avec une dette importante en raison des coûts d'études antérieures, ont dû faire face à d'importantes pressions pour continuer à travailler pour honorer leurs obligations financières. Alors que la deuxième vague de pandémie déferle actuellement pendant la saison hivernale au Canada (et que d'éventuelles vagues subséquentes se profilent aussi), ce groupe de médecins pourrait avoir à endurer une autre vague de stress et d'incertitude.

La SCA reconnaît que les anesthésiologistes ont le devoir éthique de s'efforcer de rester en bonne santé. ${ }^{17}$ Le Code d'éthique ${ }^{18}$ de l'AMC et les Lignes directrices pour la pratique éthique de l'anesthésiologie ${ }^{19}$ de l'American Society of Anesthesiologists soulignent tous les deux que les anesthésiologistes ont des responsabilités éthiques envers eux-mêmes et que la pratique de soins d'anesthésie de qualité exige qu'ils maintiennent leurs propres santé et bien-être de façon optimale. ${ }^{19}$

Nous défendons les stratégies suivantes pour protéger les anesthésiologistes et les intensivistes canadiens vulnérables (Tableau). ${ }^{15}$ L'appel à l'action de l'énoncé de position de la SCA sur la protection devrait permettre aux gouvernements, aux institutions et aux départements de soutenir et de défendre les anesthésiologistes vulnérables qui sont aux premières lignes des soins pour la COVID-19, ainsi que la santé et le bien-être globaux de chaque médecin. Des évaluations personnalisées des risques individuels devraient être réalisées ainsi que des évaluations au niveau organisationnel afin de prévenir un risque accru d'infection, de préjudice et de décès dus à la COVID-19. ${ }^{20-22}$ Il est important de tenir compte de considérations institutionnelles et départementales afin de répartir équitablement le travail, le soutien financier et l'accès à la prestation de soins cliniques sécuritaires. Pour ces travailleurs vulnérables, cela inclut la priorisation pour la vaccination, ${ }^{23,24}$ ainsi que l'augmentation, lorsque cela est possible, des options du travail à distance et de soins

\footnotetext{
A Multiple personal communciations between colleagues, departmental leadership meetings, and Ontario Medical Association Townhall Meetings during the COVID-19 pandemic.
} 
TABLEAU Mesures spécifiques pour les médecins vulnérables pendant la pandémie de COVID-19

Stratégie Action

\section{Médecins vulnérables}

Liaison et communication des risques

Modification du risque pour la santé personnelle

Actions individuelles

\section{Niveau organisationnel}

Pilier de soutien

Isolement des patients

Surveillance des risques

Actions spécifiques
Options de carrière

Dirigeants des départements et universitaire, santé au travail et ressources humaines.

-Identification selon les données les plus récentes, locales ou mondiales.

-Suivi régulier avec leur médecin traitant pour discuter de l'optimisation de leur état de santé et de stratégies de gestion des risques.

-Demande de soutien pour le bien-être mental et structure de soutien financier.

-Retour au travail dans des milieux à faible risque, p. ex., soins virtuels, chirurgies électives, remplacement d'autres collègues qui travaillent dans des milieux COVID-19, afin d'équilibrer les exigences de travail clinique et la répartition équitable du revenu.

- Travail à la maison, congé prolongé, ou congé sabbatique.

-Envisager un changement de spécialité ou une retraite anticipée pour les médecins plus âgés ou financièrement confortables.

Utilisation des interactions virtuelles ou à distance avec les patients autant que possible.

Recours à des réunions internes à distance lorsque c'est faisable.

Utilisation des EPI appropriés, des mesures de distanciation sociale et respect des mesures locales de PCI.

La vulnérabilité aux préjudices physiques augmente également la vulnérabilité aux problèmes de santé mentale et aux sentiments d'isolement et de préjudice moral. Les organismes devraient fournir tout le soutien nécessaire à ces personnes afin de permettre une répartition équitable des possibilités de revenu.

Prioriser le contact avec les patients négatifs COVID-19 et asymptomatiques pour les médecins vulnérables.

Prévenir et éliminer le contact avec les patients positifs ou ayant une suspicion de COVID-19.

$\mathrm{Y}$ compris de transmission communautaire, hospitalière et individuelle de l'infection et des effets indésirables.

Hiérarchisation des programmes de vaccination afin d'assurer une immunité contre la COVID-19.

Les médecins vulnérables ne devraient pas devoir dispenser de soins cliniques directs ou indirects aux patients positifs ou ayant une suspicion de COVID-19.

Séparer efficacement les patients en fonction de l'ampleur du risque de COVID-19.

Tester suffisamment les patients et le personnel afin de comprendre la dynamique de la COVID-19 et la prévalence de l'exposition à l'hôpital.

Assurer une adhésion stricte et continue aux politiques de masque facial par le personnel et les patients en tout temps.

Maintenir des mesures standard strictes de PCI.

Aux niveaux provincial et national

Soutien financier Création de programmes de soutien aux médecins pour fournir un soutien au plus fort des pandémies actuelle et futures.

COVID-19 = maladie coronavirus; PCI = prévention et contrôle des infections

virtuels aux patients pendant que leurs collègues effectuent d'autres travaux cliniques à haut risque. Les institutions devraient promouvoir des programmes de stabilité du revenu afin d'aider les anesthésiologistes et les autres TS à éviter toute pénalité financière majeure liée aux changements de leurs habitudes de pratique et/ou de la portée de leur pratique en raison de l'une ou l'autre des restrictions pandémiques susmentionnées et/ou de l'annulation des soins périopératoires de routine. Pour les personnes se prémunissant de la protection de proximité, les établissements devraient leur proposer un horaire de travail flexible qui tienne compte de leurs rôles de médecin, de parent et d'aidant parce que ces personnes pourraient avoir besoin d'être en congé en raison de la perte du service de garde des enfants (p. ex., en raison d'un aidant malade ou exposé à la maison, d'un parent âgé qui s'occupait des enfants, d'une fermeture d'école ou de garderie) ou en raison d'une exposition/quarantaine ou d'un test supplémentaire pour les enfants. 


\section{Les défis de la protection}

Confrontés à des défis inattendus pour leur sécurité personnelle et à l'interruption de leurs carrières et formations, les médecins britanniques se sont retrouvés confinés chez eux plus longtemps que leurs homologues, sans aucun contrôle sur le moment et la façon de retourner au travail. ${ }^{25}$ Cette déconnexion de la ligne de front a créé d'importants enjeux pratiques et psychologiques. ${ }^{25}$ Les questions liées à la divulgation volontaire de leur état de santé à leurs collègues les ont soumis à une stigmatisation et à des préjudices moraux potentiels causés par le fait de ne pas pouvoir contribuer à la réponse à la pandémie au même titre que leurs collègues (p. ex., ne pas prendre d'appels d'équipe « code bleu avec EPI » ou d'intubation). Cela les a exposés à d'autres atteintes au niveau de leur santé mentale et de leur sommeil, lesquelles ont eu un impact supplémentaire sur leur bien-être. ${ }^{26}{ }^{29}$

\section{Équité et préoccupations financières}

Lorsqu'on souhaite limiter le cadre de pratique ou du retour au travail, ces démarches doivent faire l'objet d'une planification et d'un accord coordonnés de la part des organismes nationaux, des institutions, des départements locaux et de la personne qui retourne au travail. ${ }^{10}$ Le NHS britannique et les soins de santé canadiens sont tous deux financés par l'État, mais certaines différences sont devenues apparentes à cet égard. ${ }^{30} \mathrm{La}$ réponse collaborative des médecins britanniques a abouti à une réponse plus unifiée afin de créer des systèmes d'entraide pour les médecins devant se protéger; en outre, cette cohésion a créé un environnement de soutien pour les stagiaires et les médecins spécialistes. Ces deux éléments ont servi à minimiser l'impact sur la formation et le salaire des individus en protection. La British Medical Association a déclaré qu'aucun médecin ne devait être pénalisé financièrement parce qu'il ou elle n'était pas en mesure de poser certains gestes en raison de risques posés à sa santé. ${ }^{10}$

Bien que cette stratégie de soutien ait apparemment été bien communiquée, une grande variabilité dans les expériences individuelles d'évaluation des risques et de soutien a tout de même été observée. Des comptes rendus ont fait état de pressions exercées sur les médecins pour qu'ils travaillent dans des environnements de garde inadaptés et vulnérables, ainsi que d'employeurs cherchant des façons de ne pas honorer les paiements, malgré le fait que ces personnes continuaient d'être cliniquement vulnérables. ${ }^{10}$

Comparativement au NHS britannique, le système de financement des soins de santé à payeur unique du Canada est extrêmement décentralisé (p. ex., administré par les provinces) en termes de prestation de services. De plus, les médecins canadiens sont le plus souvent des travailleurs autonomes, qui facturent les régimes provinciaux d'assurance-maladie à l'acte. ${ }^{31}$ Il n'y a que peu d'imputabilité dans les relations entre les médecins et les gouvernements, les autorités sanitaires ou les hôpitaux et ce, malgré le fait que les médecins travaillent dans le cadre des limites posées par les autorités sanitaires régionales ou provinciales. Cela s'explique en partie par le fait que les hôpitaux disposent souvent de budgets distincts et de comités indépendants, et la prise de décision est souvent fragmentée. ${ }^{32}$ Dans ce type d'environnement, il est difficile pour les anesthésiologistes canadiens de demander un soutien financier ou autre pour leur protection ('shielding').

Les efforts visant à soutenir les médecins vulnérables et les TS devraient être soupesés par rapport à la sécurité et la protection des anesthésiologistes appartenant aux catégories à faible risque, de sorte que la protection de tout le personnel d'anesthésie et des étudiants reste confidentielle, réalisée de manière équitable et non discriminatoire et ne compromette pas la sécurité des patients (Tableau). Nous sommes conscients que de telles mesures puissent être d'autant plus difficiles en raison de la pénurie actuelle de main-d'œuvre en anesthésiologie au Canada. ${ }^{33,34}$ Fait important, les principes éthiques décrits dans l'énoncé de position de la SCA s'appliquent aussi à l'ensemble des travailleurs de la santé; les TS œuvrant dans les foyers de soins de longue durée et sur les étages courent également un risque infectieux. Ceci est lié à une multitude de facteurs tels que le travail dans des fonctions faisant face ou non au patient, dans des fonctions cliniques vs non cliniques (p. ex., entretien ménager, portier), ${ }^{35}$ l'exposition professionnelle $v s$ communautaire/à la maison, le fait de travailler dans un seul endroit ou à plusieurs, la disponibilité des EPI et le respect des mesures de PCI. ${ }^{36,37}$ Bien que l'attention soit actuellement concentrée sur les soins aux personnes atteintes de la COVID-19, d'autres besoins en matière de soins de santé existent encore, notamment la nécessité de reprendre les soins chirurgicaux de routine qui mettent à rude épreuve nos ressources en soins de santé et nos TS. Afin de tenir compte de notre devoir éthique collectif envers le bien public, nous devons nous assurer de minimiser les risques encourus par nos équipes de soins de santé et d'orienter les médecins et TS vulnérables vers des milieux de travail où le risque d'infection est plus faible.

En résumé, l'appel à l'action de l'énoncé de position de la SCA appuyé par l'AMC concernant la Protection des médecins en période de pandémie ${ }^{15,16}$ propose un cadre éthique et des stratégies aux systèmes et aux institutions de santé pour répondre aux préoccupations des médecins, stagiaires et autres TS vulnérables. En unissant nos voix à 
celles d'organismes nationaux tels que l'AMC et le Centre canadien d'hygiène et de sécurité au travail (CCHST), ${ }^{38}$ des normes nationales de protection des TS devraient être établies. Un effort national, sous l'égide de la SCA et en collaboration avec l'AMC et le CCHST, devrait être déployé pour recueillir des données sur le nombre de fournisseurs d'anesthésie qui ont été infectés et, si elles sont connues, les circonstances de l'infection (p. ex., pendant les soins aux patients $v s$ ailleurs à l'hôpital ou dans la communauté). Nous préconisons que les médecins et tous les TS soient soutenus non seulement pour se sentir en sécurité et protégés contre les atteintes directes, mais également d'un point de vue moral par les dirigeants de leurs propres départements et institutions. Diverses mesures d'accommodation devraient être mises à leur disposition, y compris des options de formation et de perfectionnement à distance, une évaluation complète des risques et des plans personnalisés pour reprendre toutes les activités et/ou retourner au travail et à la formation.

Acknowledgments Mandeep Singh currently holds the Canadian Anesthesiologists' Society Career Scientist Grant and a Merit award from the Department of Anesthesiology and Pain Medicine, University of Toronto (Toronto, Canada) to support academic time. $\mathrm{He}$ also serves on the Medical Advisory Board of Hypersomnia Foundation (Atlanta, GA) at a voluntary basis.

Disclosures None.

Funding statement None.

Editorial responsibility This submission was handled by Dr. Hilary P. Grocott, former Editor-in-Chief, Canadian Journal of Anesthesia.

Déclaration Aucune.

Déclaration de financement Aucune.

Responsabilité éditoriale Cet article a été traité par Dr Hilary P. Grocott, ancien rédacteur en chef, Journal canadien d'anesthésie.

\section{References}

1. World Health Organization. Coronavirus disease (COVID-19) Situation Dashboard. Available from URL: https://www.who.int/ emergencies/diseases/novel-coronavirus-2019 (accessed February 2021).

2. Chan JF, Yuan $S, K o k K H$, et al. A familial cluster of pneumonia associated with the 2019 novel coronavirus indicating person-toperson transmission: a study of a family cluster. Lancet 2020; 395: 514-23.

3. Kamming D, Gardam M, Chung $F$. Anaesthesia and SARS. Br J Anaesth 2003; 90: 715-8.

4. Bainbridge D. Personal protective equipment (PPE) for anesthesiologists: the need for national guidelines. Can J Anesth 2020; 67: 919-23.
5. Centers for Disease Control and Prevention. Interim Infection Prevention and Control Recommendation for Healthcare Personnel During the Coronavirus Disease 2019 (COVID-19) Pandemic Available from URL: https://www.cdc.gov/ coronavirus/2019-ncov/infection-control/controlrecommendations.html (accessed February 2021).

6. Government of Canada. Infection prevention and control for COVID-19: interim guidance for acute healthcare settings. Available from URL:https://www.canada.ca/en/public-health/ services/diseases/2019-novel-coronavirus-infection/healthprofessionals/infection-prevention-control-covid-19-secondinterim-guidance.html (accessed February 2021).

7. Adams JG, Walls RM. Supporting the health care workforce during the COVID-19 global epidemic. JAMA 2020. DOI: https://doi.org/10.1001/jama.2020.3972.

8. Government of Canada. Vulnerable populations and COVID-19. Available from URL: https://www.canada.ca/en/public-health/ services/publications/diseases-conditions/vulnerable-populationscovid-19.html (accessed February 2021).

9. Public Health England; Department of Health and Social Care. Guidance on shielding and protecting people who are clinically extremely vulnerable from COVID-19. Updated: December 14, 2020. Available from URL: https://www.gov.uk/government/ publications/guidance-on-shielding-and-protecting-extremely (accessed February 2021).

10. British Medical Association. Doctors can be vulnerable too shielding under strain. Available from URL: https://www.bma. org.uk/news-and-opinion/doctors-can-be-vulnerable-tooshielding-under-strain (accessed February 2021).

11. Swan G, Watson S, Sajayan A. Supporting the shielded - results from a national survey of shielding doctors. Anaesthesia News 2020; 398: 26-7.

12. Canadian Institute for Health Information. Supply, Distribution and Migration of Physicians in Canada, 2019 - Methodology Notes. Ottawa, ON: CIHI; 2020. Available from URL: https:// www.cihi.ca/sites/default/files/document/supply-distributionmigration-of-physicians-in-canada-2019-methodology-notes-en. pdf (accessed February 2021).

13. Canadian Institute for Health Information. Physicians in Canada. Ottawa, ON: CIHI; 2020. Accessed from URL: https://www.cihi. ca/en/physicians-in-canada (accessed February 2021).

14. Canadian Institute for Health Information. COVID-19 cases and deaths among health care workers in Canada - Data Table. Ottawa, ON: CIHI; September 3, 2020.

15. McKeen D, Singh M. Canadian Anesthesiologists' Society Position Statement. Physician Shielding During A Pandemic. Available from URL: https://www.cas.ca/CASAssets/ Documents/Practice-Resources/Reports-Position-Papers/202012-CAS_ShieldingPositionStatement2.pdf (accessed February 2021).

16. McKeen D, Singh M. Canadian Medical Association Policy Endorsement. Physician Shielding During a Pandemic. Available from URL: https://policybase.cma.ca/en/permalink/policy 14386 (accessed February 2021).

17. Canadian Anesthesiolgists' Society. Practice Resources: The Healthy Anesthesiologist. 2020. Available from URL: https:// www.cas.ca/en/practice-resources/the-healthy-anesthesiologist (accessed February 2021)..

18. Canadian Medical Association. CMA Code of Ethics and Professionalism - 2018. Available from URL: https:// policybase.cma.ca/documents/policypdf/PD19-03.pdf\#_ga=2. 86141528.17093642.1606020747-94150898.1605833286 (accessed on: February 2021).

19. American Society of Anesthesiologists. Guidelines for the Ethical Practice of Anesthesiology. Available from URL: https://www. 
asahq.org/standards-and-guidelines/guidelines-for-the-ethicalpractice-of-anesthesiology (accessed February 2021).

20. Cook T, Steraton T, Watson S, et al. Workplace shielding and doctors at higher risk of harm working in anaesthesia and critical care during the COVID-19 pandemic. Accessed from URL: https://www.researchgate.net/publication/343050214_Workplace shieldinganddoctorsathigherriskofharmworkinginanaesthesiaand criticalcareduringtheCOVID-19pandemic (accessed February 2021).

21. The OpenSAFELYCollaborative; Williamson E, Walker AJ, Bhaskaran $K$, et al. OpenSAFELY: factors associated with COVID-19-related hospital death in the linked electronic health records of 17 million adult NHS patients. medRxiv. 2020. DOI: https://doi.org/10.1101/2020.05.06.20092999.

22. Cook TM. Risk to health from COVID-19 for anaesthetists and intensivists - a narrative review. Anaesthesia 2020. DOI: https:// doi.org/10.1111/anae.15220.

24. Government of Canada. Canada to receive early delivery of Pfizer-BioNTech COVID-19 vaccine. News Release, December 7, 2020. Public Services and Procurement Canada: Available from URL: https://www.canada.ca/en/public-servicesprocurement/news/2020/12/canada-to-receive-early-delivery-ofpfizer-biontech-covid-19-vaccine.html (accessed February 2021).

24. Government of Canada. Canada to receive early access to Moderna COVID-19 vaccine. News Release, December 15, 2020. Public Services and Procurement Canada: Available from URL: https://www.canada.ca/en/public-services-procurement/news/ 2020/12/canada-to-receive-early-access-to-moderna-covid-19vaccine.html (accessed February 2021).

25. Iliff HA, Simpson KA, Tomlinson CR, Webb CM; Anaesthetists, Intensivists Shielding. 'Shielded' anaesthetists and intensivists during the COVID-19 pandemic. Anaesthesia 2020. DOI: https:// doi.org/10.1111/anae.15153.

26. Alikhani R, Salimi A, Hormati A, Aminnejad R. Mental health advice for frontline healthcare providers caring for patients with COVID-19. Can J Anesth 2020. DOI: https://doi.org/10.1007/ s12630-020-01650-3.

27. Lai J, Ma S, Wang Y, et al. Factors associated with mental health outcomes among health care workers exposed to coronavirus disease 2019. JAMA Netw Open 2020. DOI: https://doi.org/10. 1001/jamanetworkopen.2020.3976.

28. Magnavita N, Garbarino S. Sleep, health and wellness at work: a scoping review. Int J Environ Res Public Health 2017. DOI: https://doi.org/10.3390/ijerph14111347.

29. Panagioti M, Geraghty K, Johnson J, et al. Association between physician burnout and patient safety, professionalism, and patient satisfaction: a systematic review and meta-analysis. JAMA Intern Med 2018. DOI: https://doi.org/10.1001/jamainternmed.2018. 3713.

30. Martin D, Miller AP, Quesnel-Vallée A, Caron NR, Vissandjée B, Marchildon GP. Canada's universal health-care system: achieving its potential. Lancet 2018; 391: 1718-35.

31. Allin S, Marchildon G, Peckham A. International Health Care System Profiles: Canada. The Commonweath Fund. June 5, 2020. Available from URL: http://international.commonwealthfund. org/countries/canada/ (accessed February 2021).

32. Government of Canada. Justice Laws Website. Canada Health Act. (R.S.C., 1985, c. C-6). Available from ULR: http://laws-lois. justice.gc.ca/eng/acts/C-6/ (accessed February 2021).

33. Orser BA, Wilson CR. Canada needs a national strategy for anesthesia services in rural and remote regions. CMAJ 2020; 192: E861-3.

34. Canadian Anesthesiologists' Society; Association of Canadian University Departments of Anesthesiology. Shortage of anesthesiologists impacts delivery of surgical, acute and chronic pain services: More residency training positions. Available from URL: https://www.cas.ca/CASAssets/Documents/About-Us/88 20-19-May-PressReleasePhysicianShortage-v2.pdf (accessed February 2021).

35. Eyre DW, Lumley SF, O'Donnell D, et al. Differential occupational risks to healthcare workers from SARS-CoV-2 observed during a prospective observational study. Elife 2020. DOI: https://doi.org/10.7554/eLife.60675.

36. Cook TM, Lennane $S$. Occupational COVID-19 risk for anaesthesia and intensive care staff - low-risk specialties in a high-risk setting. Anaesthesia 2020. DOI: https://doi.org/10.1111/ anae. 15358.

37. Tian C, Lovrics $O$, Vaisman A, et al. Risk factors and protective measures for healthcare worker infection during highly infectious viral respiratory epidemics: a systematic review and metaanalysis. Infect Control Hosp Epidemiol 2021. DOI: https://doi. org/10.1017/ice.2021.18.

38. Canadian Centre for Occupational Health and Safety. Precarious Employment and Vulnerable Workers. Available from URL: https://www.ccohs.ca/oshanswers/legisl/vulnerable.html (accessed February 2021).

Publisher's Note Springer Nature remains neutral with regard to jurisdictional claims in published maps and institutional affiliations. 